\title{
Effects of surface texture on weight perception when lifting objects with a precision grip
}

\author{
J. RANDALL FLANAGAN, ALAN M. WING, SUE ALLISON, and ANNA SPENCELEY \\ MRC Applied Psychology Unit, Cambridge, England
}

\begin{abstract}
In this paper, we show that, when lifting an object using a precision grip with the distal pads of the thumb and index finger at its sides, the perceived weight depends on the object's surface texture. The smoother the surface texture, the greater the perceived weight. We suggest that a smoother object is judged to be heavier because the grip force, normal to the surface, required to prevent it from slipping is greater. The possibility of there being an influence of surface texture per se is excluded by a second experiment that employed a variant of the precision grip in which the thumb supports the weight of the object from underneath. With the grip oriented in this way, there is no need to match grip force to surface texture and, under these conditions, there is no effect of surface texture on weight perception. In the first two experiments, the test and comparison weights were lifted successively by the same hand. In a third experiment, the effect of surface texture was replicated for sequential lifts made with separate hands. Thus, the effect is not restricted to comparisons made with the same hand.
\end{abstract}

Since Weber's (1834/1978) early studies on weight discrimination, a central issue has been whether the perception of weight depends on touch or "muscle sense." His finding that weight discrimination is improved by voluntary muscular exertion suggests that we should be concerned with the contribution of a "sense of force" to weight perception (Bell, 1834). Our interest in this paper is in contributions to weight perception of muscle activity that is only indirectly related to that supporting the weight.

Many studies of weight perception concur on the role of muscle activation in weight discrimination (for a review, see Jones, 1986). While there is debate about the relative importance of efferent and afferent muscle signals (see Matthews, 1982), it is normally implicitly assumed that the relevant muscles are those directly involved in lifting and supporting the judged load against gravity. However, Kilbreath and Gandevia (1991) have recently reported that the perceived heaviness of a reference weight lifted by one finger increases if a concurrent weight, greater than or equal to the reference weight, is lifted at the same time by another finger of the same hand. Thus, perceived weight may depend on muscle activity not directly involved in lifting. However, these authors also observed that if the concurrent weight was lifted by the ankle, there was no effect. This suggests that the bias associated with a concurrent weight depends on whether the muscle forces involved are functionally related in everyday tasks, as might reason-

We thank Ian Nimmo-Smith for help with the statistical analysis. J. R. Flanagan is now at the Department of Psychology, Queen's University, Kingston, Ontario. Correspondence should be addressed to A. M. Wing, Medical Research Council, Applied Psychology Unit, 15 Chaucer Rd., Cambridge CB2 2EF, England (e-mail: a.m.wing@mrcapu.cam.ac.uk) ably be assumed to be the case for the muscle forces exerted by one hand.

Weight discrimination experiments are often performed with the hand supporting the object from below (it having been placed there by the experimenter). However, when lifting an object from a surface, it is common to grasp it from the side or from above, with the thumb on one side and one or more fingers on the other side. In this case, frictional force counteracts the load during the lift. This force arises from the forces, normal to the object sides, developed by the digits.

To prevent slip, the limiting friction $(f)$ between the object and the skin must be greater than or equal to shear force due to the load. The limiting friction depends on the coefficient of static friction $(\mu)$ between the skin and the touched surface and the grip force $(G)$ :

$$
f=\mu G .
$$

To successfully lift an object, the grip force must be greater than a minimum, below which slip would occur. For a given weight, this minimum will depend on the surface texture; a smooth texture is typically more slippery in that its coefficient of friction is lower than that of a rough surface. Johansson and Westling (1984) have shown that when holding an object using a precision grip (with the distal pads of the thumb and index finger at its sides), grip force is typically just slightly greater than this minimum (see Figure 1). In other words, the grip force is scaled to the weight of the object and its surface texture; the heavier the object and the more slippery the surface, the greater the grip force.

Depending on the task conditions, the scaling of grip force may be predictive. For example, the subject may be given a number of trials with a particular set of objects so that he/she becomes familiar with the weight. In such 

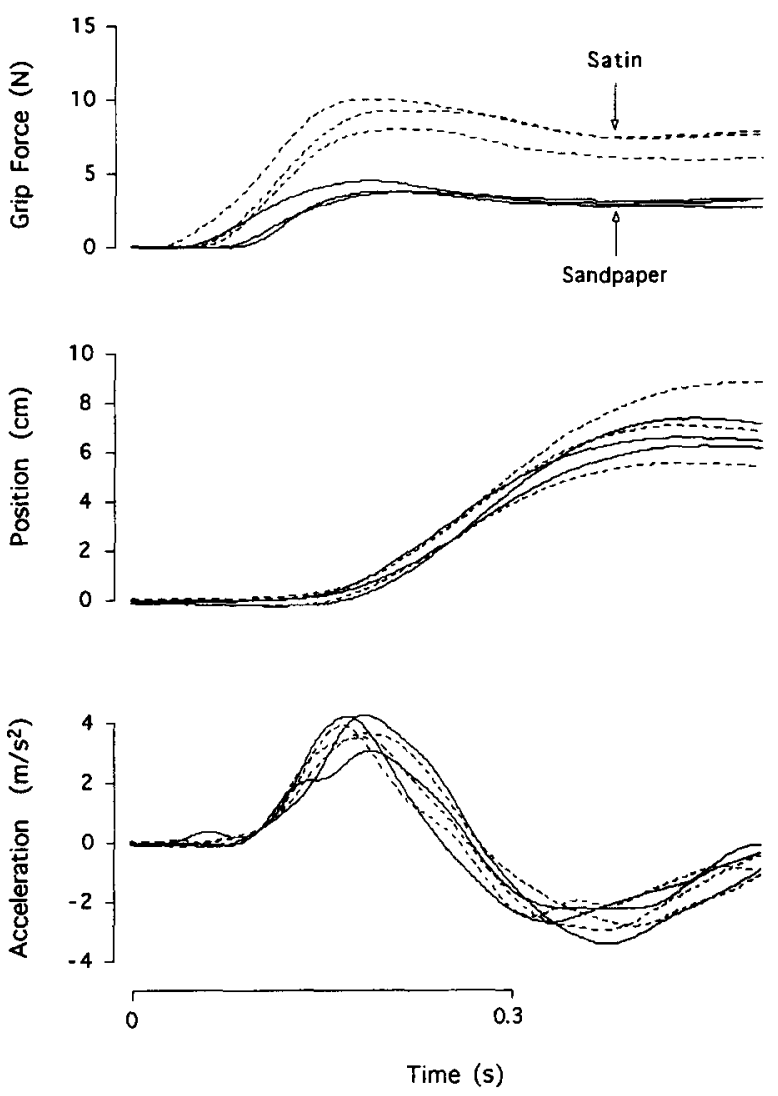

Figure 1. Changes in grip force with surface slipperiness when lifting a force transducer (mass $=0.26 \mathrm{~kg}$ ) using a precision grip. This figure shows data from our laboratory of form similar to those published by Johansson and Westling (1984). The top, middle, and bottom panels show grip force and the vertical position and acceleration of the transducer. The dashed traces show individual trials in which the contact surfaces were covered with slippery satin; the solid traces show trials in which the contact surfaces were covered in less slippery sandpaper. Although the position and acceleration functions in the "satin" and "sandpaper" trials are similar (so that the load forces are approximately equal), the grip force rises more rapidly and reaches a greater final level when the surface is more slippery.

cases, even before an object is lifted clear of its support surface and before tactile information can be used to adapt grip force, the rate of rise of grip force anticipates the final value required by the object's weight (Johansson \& Westling, 1984). Moreover, Gordon, Forssberg, Johansson, and Westling (1991a, 1991b) have shown that the scaling of grip force to weight may be based on visual cues. When object size changes from trial to trial, subjects select greater initial rates of rise of grip force with larger objects than with smaller objects. Nonetheless, if the prediction turns out to be incorrect (perhaps because it was falsified by experimental subterfuge), concurrent sensory information may be used to make secondary adjustments to the initial selection of target grip force (Johansson \& Westling, 1988).

In our own work, we have shown that grip force is modulated with the fluctuations in inertial load that arise from moving an object (Flanagan, Tresilian, \& Wing, 1993; Flanagan \& Wing, 1993). As the object is accelerated then decelerated by the arm, depending on the direction of motion relative to gravity, the total load may considerably exceed the weight (i.e., the load due to gravity). In such situations, a rise in grip force coincides with the increase in load; the correspondence in timing indicates that the grip force modulation is an anticipation of (rather than a reaction to) the self-induced load force. We have also shown that the extent of modulation increases with more slippery surfaces (Flanagan \& Wing, 1994).

The finding that grip force scales with load and surface texture in the lifting, holding, and moving tasks may have implications for weight judgments. Because grip force is related to load force, grip force may be a useful cue for discriminating weight. However, discrimination could be confounded if the surface textures of the weights to be discriminated are different, requiring differences in grip force. In this paper, we look at effects of surface texture during lifting with a precision grip, with the distal pads of the thumb and index finger at the sides of the object. By varying the surface, we manipulated the grip force independently of the load force and thus could look for possible effects of grip force on perceived load. We hypothesized that the additional grip force used to grasp a more slippery object would influence the perceived load. In particular, we predicted that subjects would perceive a slippery object to be heavier than a less slippery object of the same weight because of a failure to distinguish between the additional normal forces required to grip the slippery object and the shear forces, which will be the same regardless of surface.

We report the results of three experiments. In the first experiment, subjects had to compare the weight of a test object with the weight of a reference object after lifting each in turn, using a vertical precision grip with the digits at the sides (Figure 2A). In the second experiment, subjects held each object in either a vertical grip or a horizontal grip with the digits above and below the object (Figure 2B). In the horizontal grip, there is little or no shear force tending to slide the object from the grasp, so grip force is not needed to generate an opposing frictional force. Thus, changing the surface texture of objects held in this grip allowed an examination of whether there was an effect of surface texture, per se, on the perception of weight. In the third experiment, subjects lifted test and comparison objects successively, as in the first two experiments, but with separate hands. The aim was to test whether any possible effects of surface texture on perceived weight would be restricted to weights judged by lifting with one hand or whether the effects would generalize to between-hand judgments.

\section{Method}

\section{EXPERIMENT 1}

Subjects. Forty subjects, including members of the subject panel and staff of the MRC Applied Psychology Unit, participated in this 

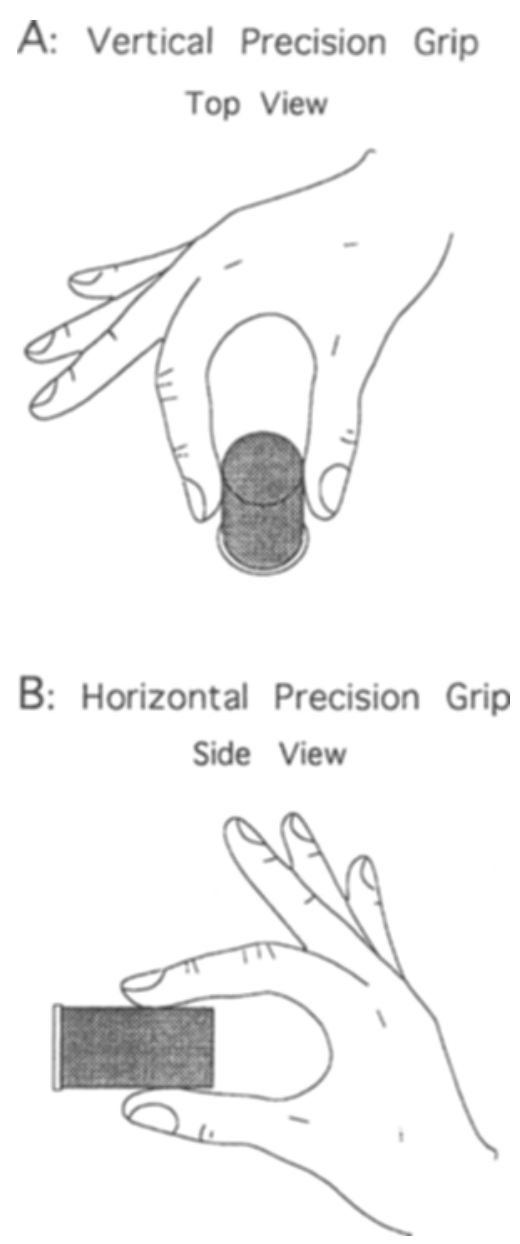

Figure 2. (A) Vertical precision grip: Friction forces generated by grip force at right angles to the canister's surface counteract the weight. (B) Horizontal precision grip: The weight is supported by the thumb, with the index finger providing a very light force to prevent the canister from rolling sideways. To vary the surface texture, the canister was wrapped in either sandpaper or satin.

experiment with informed consent. The panel subjects were paid for their participation. The subjects included 20 males and $20 \mathrm{fe-}$ males, and their ages were distributed evenly in the range 18 to 65 years. Two subjects who reported tactile difficulties were excluded, and one other subject was excluded because she was unable to discriminate between weights even when the difference was large. The subjects were told that they would have to compare the weight of a series of test objects with the weight of a reference object and that the same reference object would be used throughout the series.

Stimuli. The objects consisted of $35-\mathrm{mm}$ film canisters filled with a variable number of coins (tuppenny pieces). The canisters, which were $50 \mathrm{~mm}$ long and $30 \mathrm{~mm}$ in diameter, were covered by either satin or coarse-grain sandpaper to give a smooth or rough surface. The average coefficient of friction between the skin and surface was about 0.5 for satin and 1.1 for sandpaper (Flanagan \& Wing, 1994). There were nine "test" canisters for each surface texture, ranging from 80.1 to $151.1 \mathrm{~g}$ in increments of $7.1 \mathrm{~g}$, with the exception of the lightest and heaviest canisters, whose increments were $14.2 \mathrm{~g}$. In addition, there were two "reference" canisters, one for each texture, with a mass of $115.6 \mathrm{~g}$. This corresponds to the central value of the test canisters.

Procedure. Each subject completed four sets of nine trials. In each set, they had to compare the weights of the nine test canisters with the weight of a reference canister. At the start of each trial, the experimenter placed the reference canister and one of the test canisters on a bench in front of the subject, who was seated. The order in which the test canisters were presented was randomized. In all trials, the subject first lifted the reference canister and then lifted the test canister. The subject's task was to tell the experimenter whether the test canister was lighter than the reference, heavier than the reference, or equal in weight to the reference. However, the subjects were encouraged to respond "lighter" or "heavier" if possible. The subjects were allowed to repeat the procedure if they were not sure whether the test canister was lighter or heavier. In most instances, the subjects responded after a single lift of each object.

The subjects grasped the canister using a precision grip with the distal pads of the thumb and index finger at the sides (see Figure $2 \mathrm{~A}$ ). This grip was demonstrated by the experimenter at the start of the session. The subjects always lifted the canister with the same, preferred hand. Typically, the subjects held the canister for 2 or $3 \mathrm{sec}$ before replacing it on the bench. They were permitted to move it up and down, and many subjects did do this once or twice during each lift.

In the first two sets of trials, the surface textures of the reference and test canisters were different. In the "smooth-rough" trials, the surface of the reference canister was smooth and that of the test canisters was rough; in the "rough-smooth" trials, the reference canister was rough and the test canisters were smooth. Half of the subjects (Group 1) started with the smooth-rough trials; the other half (Group 2) started with the rough-smooth trials. In the last two sets of trials, the surface textures of the reference canister and test canister were the same, that is, "smooth-smooth" or "rough-rough." Half of the subjects in each of the two groups started with the smooth-smooth trials; the others started with the rough-rough trials.

Analysis. For each of the four conditions (i.e., smooth-rough, rough-smooth, smooth-smooth, rough-rough), the probability of responding "lighter," $p(\mathrm{~L})$, or "equal," $p(\mathrm{E})$, was determined for each test canister. The probability is simply the ratio of the number of subjects who responded "lighter" (or "equal") divided by the total number of subjects (i.e., 40). The probability was then plotted as a function of the mass of the test canister. Logit analysis (McCullagh \& Nelder, 1989) was used to test for differences in the probability functions between conditions.

\section{Results and Discussion}

Figure 3 shows $p(\mathrm{~L})$ (top panel) and $p(\mathrm{E})$ (bottom panel) when the reference and test canisters were either both smooth (open circles) or both rough (solid circles). The following logit model was used to obtain the estimated $p(\mathrm{~L})(\pi)$ as a function of the mass of the test canister and surface texture:

$$
\pi=\frac{\exp \left(\beta_{0}+\beta_{1} T+\beta_{2} \text { Mass }\right)}{1+\exp \left(\beta_{0}+\beta_{1} T+\beta_{2} \text { Mass }\right)}
$$

where the dummy variable $T$ codes for the texture $(T=0$ for smooth and $T=1$ for rough). This model assumes that the probability functions of the two textures are equal in steepness but that one function may be shifted, left or right, with respect to the other. As can be seen in 

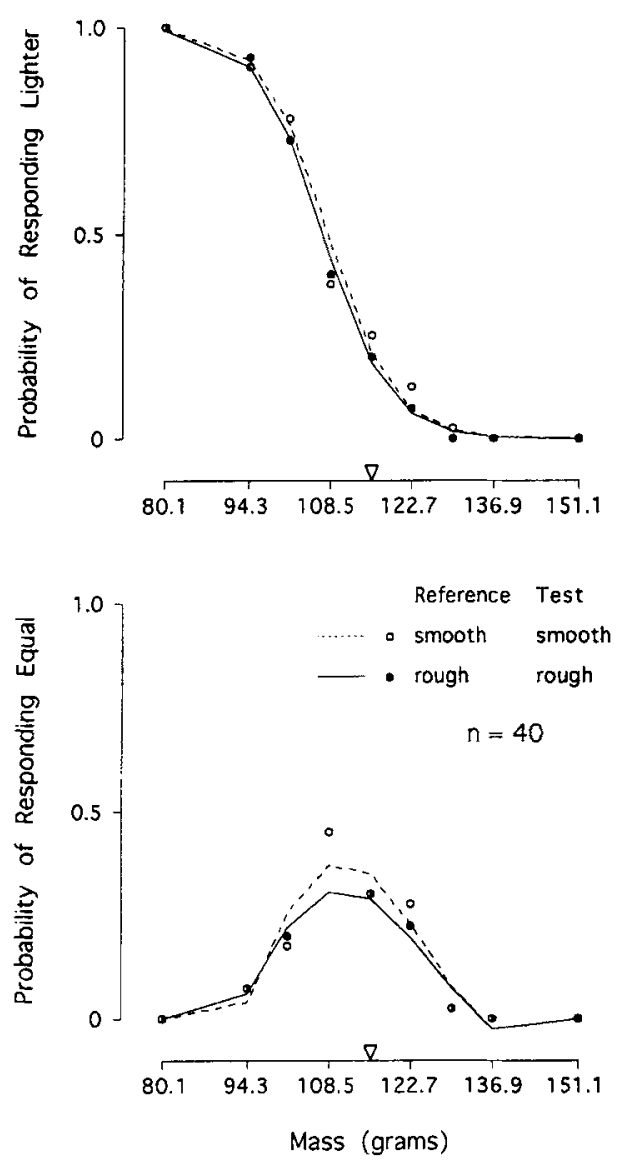

Figure 3. Group $(n=40)$ psychometric functions for weightdiscrimination judgments when test and reference canisters were either both smooth or both rough. Top: Probability of responding test canister is lighter than the previously lifted reference canister. Bottom: Probability of saying test and reference canisters are of equal weight.

the figure, this model provides a good fit to both the smooth-smooth and rough-rough data. The values of $p(E)$ of each texture were fit with a quintic polynomial.

At any given test mass, the values of $p(\mathrm{~L})$ for the two surface textures are similar. For both textures, $p(\mathrm{~L})$ is 1 for the lightest test mass and 0 for the heaviest test mass. A strong bias toward saying that the test canister was heavier was observed. When the test canister was the same mass as the reference $(115.6 \mathrm{~g}$-indicated by the open arrow), $p(\mathrm{~L})$ was 0.25 when the surface textures were smooth and 0.20 when the surface textures were rough.

The $p(\mathrm{~L})$ curves for the two surface textures are similar in form. Moreover, the $p(\mathrm{E})$ curves are also similar. As would be expected, the $p(\mathrm{E})$ function is an inverted $\mathrm{U}$.

Figure 4 shows the $p(\mathrm{~L})$ and $p(\mathrm{E})$ when the surfaces of the reference and test canisters were different (smoothrough or rough-smooth).

Logit analysis was used to test for differences in the probability of responding "lighter" for the smooth-rough and rough-smooth conditions. The following model was evaluated (which is a transform of Equation 2):

$$
\log \left\{\frac{p \mathrm{~L}}{1-p L}\right\}=\beta_{0}+\beta_{1} T+\beta_{2} \text { Mass, }
$$

where the dummy variable $T$ codes for condition $(T=0$ for rough-smooth trials and 1 for smooth-rough trials). The analysis showed that $\beta_{1}$ was reliably greater than zero $[t(17)=5.41, p<.001]$. This indicates that the "shift" to the right of the probability function obtained when the test canister was smooth is significant and confirms what is clearly apparent in Figure 4-that the probability of saying that a given test canister is "lighter" than the reference is significantly less if the canister is covered in the more slippery surface. This suggests that when an object's surface requires a greater grip force, it is perceived as heavier. A similar analysis to compare the curves in Figure 3 for rough-rough and smooth-smooth conditions yielded a nonsignificant result $[t(17)=0.63, p>.5]$.
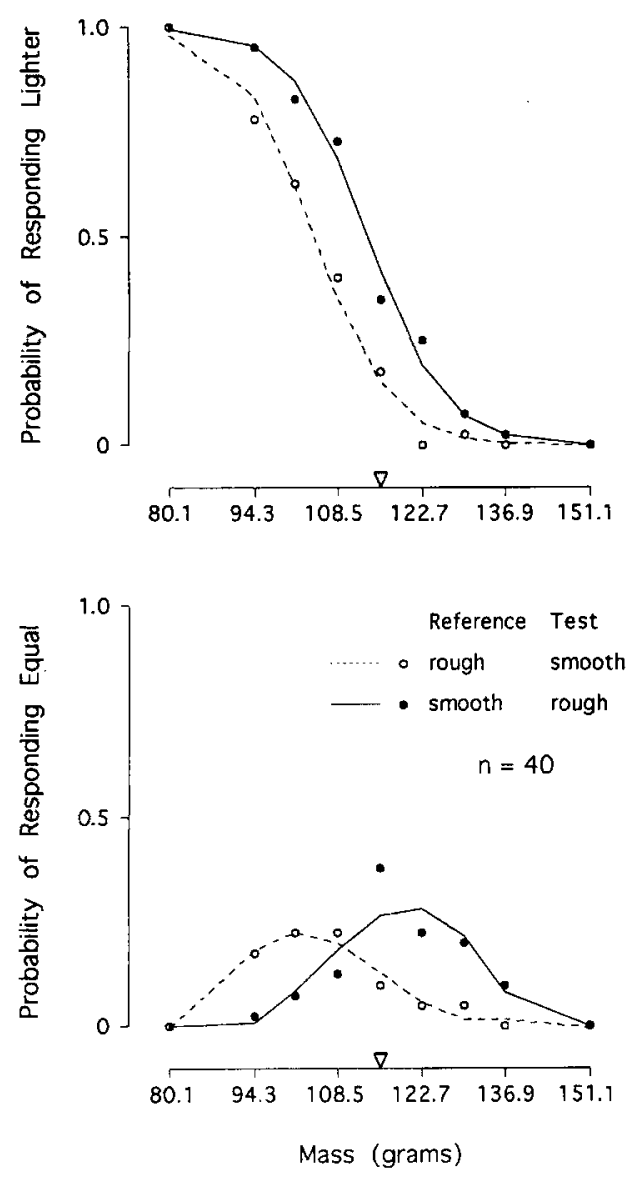

Figure 4. Group ( $n=40)$ psychometric functions for weightdiscrimination judgments showing effect of differences in surface texture. Top: Probability of responding that the test canister is lighter than the previously lifted reference canister. Bottom: Probability of saying that the test and reference canisters are of equal weight. 
In logit analysis, for any given value of $p(\mathrm{~L})$, the difference between the estimated masses of the rough and smooth test canisters is given by the ratio of $\beta_{1}$ to $\beta_{2}$. We found this difference was $9.0 \mathrm{~g}$. In other words, on average, the mass of the rough test canister had to be $9.0 \mathrm{~g}$ greater than the mass of the smooth object for $p(\mathrm{~L})$ to be perceived as being the same. Note that this is about $30 \%$ greater than the smallest difference in mass between test canisters.

We found that there was no effect of the order in which the rough and smooth canisters were presented. That is, the effect of texture did not depend on whether the smooth or the rough canister was the reference. We were originally concerned that the grip force used to hold a rough test canister following a smooth reference might be maintained at the (higher) level appropriate for the smooth reference. With equal grip forces, there would have been no reason to expect an effect of texture on perceived weight. (Note that in going from a rough reference to a smooth test grip force must increase to avoid slip.) The fact that no order effect was found suggests that the subjects did adjust their grip force on each lift. This would be consistent with the results of Johansson and Westling (1988), who have shown that when surface texture is changed after a set of trials with a particular texture, the grip force is rapidly adapted to the new texture and that this occurs even before the object is lifted off the bench. By the time the full weight of the object has been taken up by the hand, the grip force-which may be initially influenced by the grip force employed on the previous lift-is completely adapted to the new texture. Thus, an order effect would not be predicted.

\section{EXPERIMENT 2}

We have inferred from the results from Experiment 1 that the magnitude of the grip force used to hold an object influences its perceived load. Smooth objects are judged to be heavier because higher grip forces are required to hold them (Johansson \& Westling, 1984). However, it is possible that the effect we observed was due to texture per se. To exclude the possibility that the results of Experiment 1 were due to texture alone, subjects were asked to judge weights using a horizontal grip (see Figure 2B) in which the weight of the object is supported from below by the thumb. (The thumb was chosen because Kilbreath and Gandevia, 1991, have reported that the muscles of the thumb are more accurate in estimating weight than are the muscles of the index finger.) With this horizontal grip, very little grip force is required to hold the object, since frictional forces are not required to counteract the weight of the object. Thus, the grip force required for the smooth and rough objects is the same. Therefore, if the effect of texture in Experiment 1 was due to grip force and not to surface texture per se, there should be no difference in judgments of the weight of smooth and rough objects held in the horizontal grip.
In this second experiment, subjects were asked not to move the test canister when judging its weight; the experimenter placed it between the subject's thumb and finger at the beginning of the trial and removed it at the end. The subject held the canister aloft with the arm unsupported. Because of this change in testing procedure, we also tested the vertical grip in Experiment 2.

\section{Method}

Subjects. Fourteen subjects from the Applied Psychology Unit subject panel were paid to participate in this experiment. None of these subjects had participated in Experiment 1. All of them reported that they had no sensory or motor problems.

Stimuli. The objects used for weight judgment were the same canisters that were used in Experiment 1.

Procedure. The procedure was similar to that of Experiment 1, except that the canisters were given to the subject to hold in a constant position. That is, the subject did not lift the canisters from the bench. In each trial, the experimenter first placed one of the nine reference canisters in the subject's grasp and then, after about $3 \mathrm{sec}$, removed this and replaced it with the test canister. After a further $3 \mathrm{sec}$, the test canister was removed and the subject was asked to say whether the test was heavier, lighter, or equal in weight to the reference canister. As in Experiment 1, the subjects were encouraged to respond "heavier" or "lighter" if possible. The experimenter placed and removed the canister by holding it and waiting for the subject to close or open his/her digits, respectively. Thus, the canister was neither pulled nor pushed while in the subject's grasp since this would have produced horizontal loading.

In the horizontal grip, the canister was grasped in the center so that the torques and lateral forces acting at the point where the thumb contacted the canister were minimal. As a result, the grip force required to stabilize the canister would have been very small in comparison with that required by the vertical grip.

Each subject completed four sets of nine trials, two sets for each grip. All subjects performed the horizontal grips first, followed by the vertical grips. For both grips, the order of the surface textures was randomly varied across subjects. Thus, for some subjects, the reference was smooth in the first set (smooth-rough) and rough in the second (rough-smooth); for others, the order was the opposite. Note that we only examined the case where the surface textures of the reference and test canisters were different.

\section{Results and Discussion}

Figure 5 shows $p(\mathrm{~L})$ curves obtained when the canister was held with either the vertical (top panel) or the horizontal (bottom panel) grip. Data shown for the roughsmooth (open circles, dashed line) and smooth-rough (solid circles, solid line) conditions are shown in both panels. As can be seen in the top panel, the main result from the first experiment was replicated in the present experiment. That is, when holding an object with a vertical grip, the perceived load is greater when the surface is smooth. In contrast, the bottom panel shows that when an object is held with a horizontal grip, the perceived weight does not depend on surface texture. Figure 5 also shows that the slope of the probability curve is greater for the vertical grip than for the horizontal grip. This indicates that the sensitivity to perceived load is greater when an object is held with the vertical grip, where shear forces act on the skin contact area. 


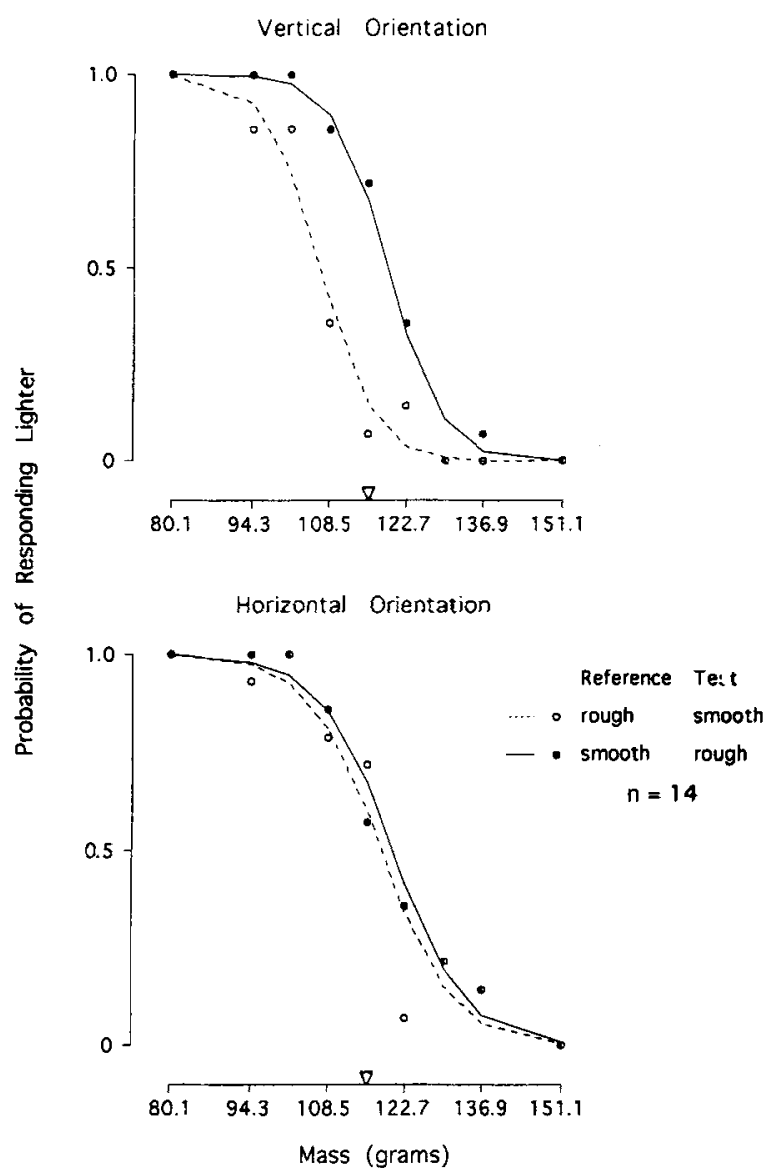

Figure 5. Group $(n=14)$ psychometric functions for weightdiscrimination judgments showing an effect of difference in surface texture on probability of saying that the test canister is lighter than the reference when using the vertically oriented precision grip (top) but not the horizontally oriented precision grip (bottom).

Logit analysis was used to test for differences between the smooth-rough and the rough-smooth conditions for both grips (see Equation 3). For the vertical grip, the difference in the intercept $\left(\beta_{1}\right)$ was found to be significant $[t(17)=4.49, p<.001]$. The difference was $12.3 \mathrm{~g}$, which is slightly larger than the difference obtained in the first experiment $(9.0 \mathrm{~g})$. Thus, on average, the mass of the rough test canister had to be $12.3 \mathrm{~g}$ greater than the mass of the smooth test canister for $p(\mathrm{~L})$ to be the same. In contrast, for the horizontal grip, there was no reliable difference $[t(17)=0.79, p>0.2]$.

In conclusion, the results from this experiment support the hypothesis that differences in perceived weight between smooth and rough objects held in a vertical precision grip reflect differences in grip force required to prevent slip and are not due to texture per se.

\section{EXPERIMENT 3}

Gordon, Forssberg, and Iwasaki (1994) have recently suggested that sensorimotor memory for object weight transfers from one hand to the other. Their experiment involved lifting a series of objects of varying weight, using either one hand or two hands in alternation. The weight of the object could not be predicted from visual cues. In the one-hand condition, they reported that the rate of rise of grip force depended on the weight of the previously lifted object (see also Johansson \& Westling, 1988). This was also true in the two-hand condition. Thus, if weight discrimination in sequential lifting is based on sensorimotor memory, then the bias demonstrated in the first two experiments, in which the canisters were lifted with one hand, might also be expected when lifting the reference canister with one hand and the test canister with the other. In this experiment, we assessed whether the effect of surface texture on perceived weight would be observed when the canisters were lifted successively but with different hands. A question that arises is whether the comparison of forces with those stored in memory is similar when comparing across hands, as it is when comparing within a hand.

\section{Method}

Subjects. Eleven graduate students from the Applied Physiology Program at Teachers College, Columbia University received extra course credit for participating in this experiment. All of the subjects were naive with respect to the experimental hypothesis and reported that they had no sensory or motor problems. All of the subjects were right-handed. One of the subjects only completed the conditions in which the surface textures of the test and reference canisters were different.

Stimuli. The objects used for weight judgment were the same canisters used in Experiments 1 and 2.

Procedure. The procedure was similar to that of Experiment 1, except that the canisters were lifted by alternate hands. That is, the subject first lifted the reference object with one hand and then, after having replaced the reference object, lifted the test object with the other hand. The time delay between lifts was approximately the same as in Experiment 1. Five subjects always used their right hands to lift the reference canister and 6 subjects always used their left hands. Each subject first completed the two conditions in which the surface textures were the same and then completed the two conditions in which the surface textures were different. The order of conditions (rough-rough vs. smooth-smooth or rough-smooth vs. smooth-rough) was varied across subjects.

\section{Results and Discussion}

Figure 6 shows $p(\mathrm{~L})$ curves for the smooth-smooth and rough-rough conditions (top) and the smooth-rough and rough-smooth conditions (bottom). As can be seen in the top panel, the $p(\mathrm{~L})$ curves for the smooth-smooth condition (open circles, dashed line) and the roughrough condition (solid circles, solid line) are very similar. Thus, when the surface texture of the reference and test canisters is the same, there is no effect of texture on perceived weight. Note that the bias toward responding that the (second) test canister is heavier than the reference canister, observed in the first two experiments, is again evident.

In the bottom panel, the $p(\mathrm{~L})$ curve for the smoothrough condition (solid circles, solid line) can be seen to be shifted to the right relative to the $p(\mathrm{~L})$ curve for the rough-smooth condition (open circles, dashed line). 


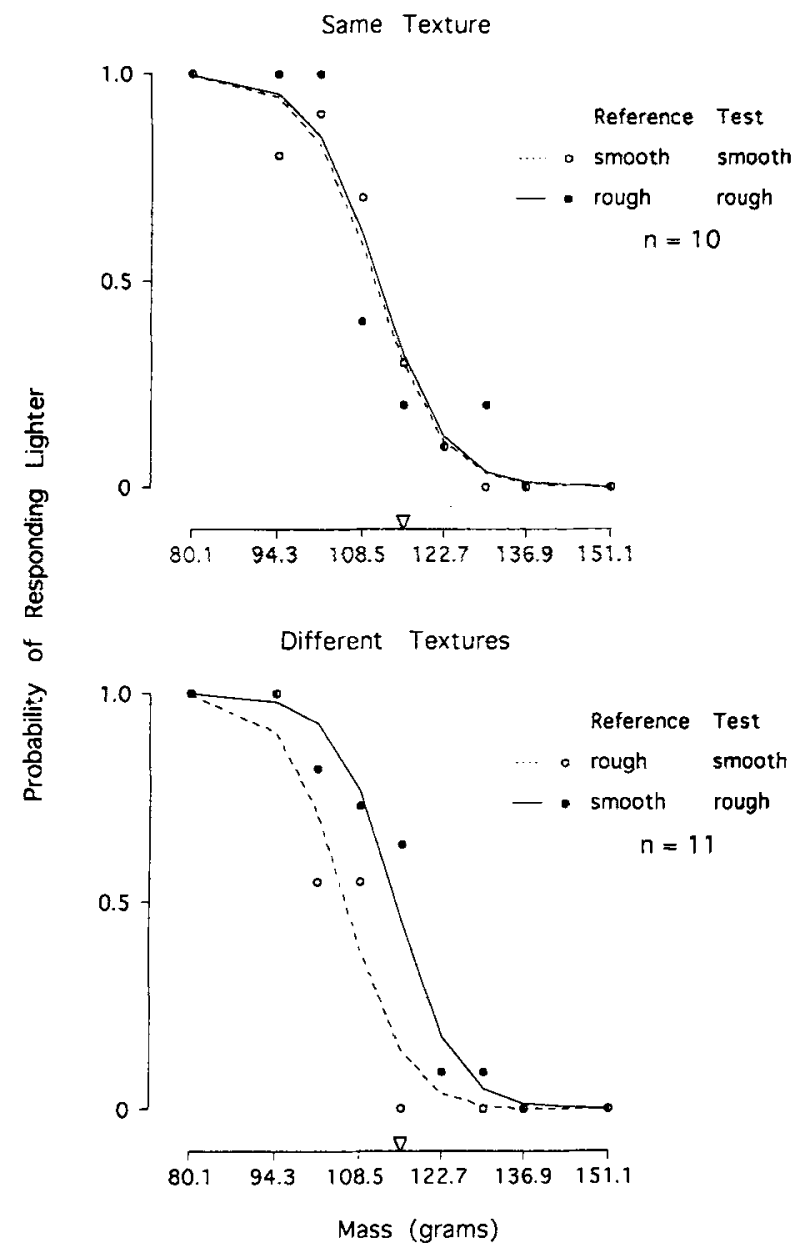

Figure 6. Group psychometric functions for weight-discrimination judgments when lifting the test and reference canisters with separate hands. (One of the 11 subjects only completed the conditions in which surface textures differed.) Top: Probability of responding test canister is lighter than the previously lifted reference canister when the surface textures of the canisters are the same. Bottom: Probability of responding test canister is lighter than the previously lifted reference canister when the surface textures of the canisters are different.

This indicates that for a given test weight, the smooth object was judged to be heavier than the rough object.

These observations were confirmed by logit analysis. The difference in the intercepts $\left(\beta_{1}\right)$ between the smoothrough and the rough-smooth $p(\mathrm{~L})$ curves was statistically significant $[t(17)=2.98, p<.01]$. The difference was $8.6 \mathrm{~g}$, which is just slightly less than the difference obtained in Experiment 1 (9.0 g). By comparison, the difference in intercepts $\left(\beta_{1}\right)$ between the smoothsmooth and the rough-rough curves was less than a gram and was not reliable $[t(17)=0.26, p>.5]$.

In conclusion, the results demonstrate that the effect of surface texture on perceived weight is observed both when the subject lifts successively with different hands and when lifting with the same hand (see Experiments 1 and 2).

\section{GENERAL DISCUSSION}

In the three experiments reported in this paper, we have shown that when subjects use a vertical precision grip (with the thumb and index finger on either side) to compare the weights of two objects of the same size lifted in succession, discrimination of their weight is influenced by their surface texture. If two objects of equal weight are being compared and one has a smooth surface and the other a rough surface, the smooth-surfaced object is likely to be judged as being heavier than the object with the rough surface.

In the second experiment, we showed that the bias in weight perception is not a function of the surface texture per se. When subjects use a horizontal precision grip in which the weight of the object is supported by the thumb underneath, there is no effect of surface texture on perceived weight. This suggests that the tendency to judge a smoother object as being heavier than a rough object of the same weight may arise from the fact that higher grip forces are required with a smooth object held in a vertical precision grip to prevent it from slipping than are required with a rough object (Johansson \& Westling, 1984). However, other interpretations are possible. For example, the rate of discharge of cutaneous afferents may be greater for smooth objects than for rough objects when the lifting is done with a vertical grip (where shear force is significant) but not when it is done with a horizontal grip. If this were the case, the effect of texture on perceived weight when lifting with a vertical grip could reflect cutaneous afferent information.

One interpretation of our results is that subjects do not fully distinguish between the normal force used to grip the object and the frictional force used to counter the load. It may be that the normal forces exerted by the thumb and index finger in gripping the object's sides constitute a "concurrent load" in the sense of Kilbreath and Gandevia (1991). These authors showed that perceived heaviness of a load supported by one finger is greater if another finger of the same hand also supports a load. Our findings may represent a functional version of this phenomenon. In this respect, it is interesting to note that Kilbreath and Gandevia reported that the perceived weight increased only if the concurrent weight was greater than or equal to the reference weight. Thus, the question arises as to whether--in our task - the grip force $(G)$ exerted by each digit is greater than or equal to the load or shear force $\left(F_{\mathrm{s}}\right)$ on each digit.

The minimum grip force required to prevent slip $\left(\mathrm{G}_{\min }\right)$ is $F_{\mathrm{s}} / \mu$, where $\mu$ is the coefficient of static friction between the skin and the touched surface (see Equation 1). Thus, if $\mu$ is equal to unity, $G_{\min }$ will be equal to $F_{\mathrm{s}}$. In other words, the minimum grip force that must be applied by each digit will be equal to the load or shear force acting on the two digits (or twice the load force acting on a single digit). If $\mu$ is less than unity, $\mathrm{G}_{\min }$ will be greater than $F_{\mathrm{s}}$ and if $\mu$ is greater than unity, $\mathrm{G}_{\min }$ will be less than $F_{\mathrm{s}}$. 
In a previous report (Flanagan \& Wing, 1994), we estimated, for 4 subjects, values of $\mu$ for the sandpaper and satin surfaces used in the present study. The values of $\mu$ for sandpaper ranged from 0.92 to 1.33 . (The individual differences in $\mu$ may reflect variation in factors such as dryness of skin.) This suggests that when holding a rough (sandpaper) object, $\mathrm{G}_{\min }$ will be roughly twice the load force acting on a single digit. The values of $\mu$ for satin ranged from 0.31 to 0.76 . This indicates that when holding a smooth (satin) object, $\mathrm{G}_{\min }$ will be considerably greater in magnitude than the load force on each digit. Moreover, the actual grip force used will be slightly greater than the minimum (Johansson \& Westling, 1984). Thus, it is reasonable to suppose that, in our task, the grip forces were, in general, greater than the shear forces acting on the digits. Thus, our results were obtained under the conditions described by Kilbreath and Gandevia (1991). In future work it would be interesting to look at rougher, even sticky, surfaces, for which the grip force can be appreciably less than the shear force, to see whether a bias still obtains. If so, this would represent a departure from the restriction described by Kilbreath and Gandevia for concurrent loads.

It is likely that when lifting with a precision grip, some hand muscles (e.g., first dorsal interosseus) will contribute to both grip force and load force. It is possible that this may partly explain the bias of grip force on perceived load. However, the results of Kilbreath and Gandevia (1991) do not support this view. They reported similar increases in perceived weight when lifting test and concurrent weights with two portions of the same muscle (flexor digitorum profundus) as when lifting with muscles that are functionally related but have anatomically distinct tendons (flexor pollicus longus and the index portion of flexor digitorum profundus).

Johansson and Westling (1988) have suggested that anticipatory scaling of grip force to the weight and surface texture of an object is based on a sensorimotor memory that is built up from previous experience. If this memory were used in successive weight discriminations, we might expect weight judgments to be unbiased by surface texture. The fact that there is a bias might suggest that the weight- and texture-related components of this memory are not dissociable or that some other information forms the basis of the judgment. For example, the effort used to grip the object may be the relevant cue. In the first two experiments, subjects lifted the test and reference objects with the same hand. In the third experiment, we showed that the effect of surface texture on weight perception is also observed when the subject lifts with one hand and then the other, in succession. This suggests that sensorimotor memory obtained from lifting with one hand is available for comparison with information subsequently obtained when lifting with the other hand, and is consistent with the recent finding of Gordon et al. (1994) described earlier.

The memory hypothesis suggests future studies to examine the stability of the stored representation over time. These might include studies in which the delay between lifts of the reference and test canisters is manipulated and studies in which instructions are given to the subject for attention to be focused on various available cues. For example, the subject could be informed about the coupling between grip force and surface slipperiness or told to focus on the effort exerted by arm muscles.

The present results may be contrasted with the findings of Gordon et al. (1991a, 1991b), who studied the effects of the size-weight illusion (Charpentier, 1891; Ross, 1969) on precision grip force. They reported that the rate of rise of grip force is greater when lifting larger objects whose weights are perceived to be less than those of smaller objects of the same weight. Subjects perceive a larger object to be lighter even though the grip force used to grasp it is the same. In contrast, we find that subjects judge smooth objects, which require greater grip forces, to be heavier than rough objects of the same weight. Thus, it would appear that any effect of grip force on perceived weight may be overridden by (at least some) higher level mechanisms such as those underlying the size-weight illusion.

The resolution of some of the questions raised in this discussion define interesting areas for further research. However, by way of conclusion, we restate the primary finding of the present study. The surface texture of objects influences the perception of their relative weights when the objects are lifted in succession with a precision grip. The effect may be attributed to the elevation of grip force that is required to prevent the more slippery object from slipping. Thus, the automatic and tight coupling between grip and load force seen in studies of motor control has perceptual consequences.

\section{REFERENCES}

BeLL, C. (1834). The hand. Its mechanism and vital endowments as evincing design. London: Pickering.

Charpentier, A. (1891). Analyse expérimentale des quelques éléments de la sensation de poids [Experimental study of some aspects of weight perception]. Archives de Physiologie Normales et Pathologiques, 3, 122-135.

Flanagan, J. R., Tresilian, J. R., \& Wing, A. M. (1993). Coupling of grip force and load force during arm movements with grasped objects. Neuroscience Letters, 152, 53-56.

FlanaGan, J. R., \& WING, A. M. (1993). Modulation of grip force with load force during point to point arm movements. Experimental Brain Research, 95, 131-143.

Flanagan, J. R., \& Wing, A. M. (1994). Coordination of grip force with load force in cyclic arm movements. Manuscript submitted for publication.

Gordon, A. M., Forssberg, H., \& Iwasaki, N. (1994). Formation and lateralization of internal representations underlying motor commands during precision grip. Neuropsychologia, 32, 555-568.

Gordon, A. M., Forssberg, H., Johansson, R. S., \& Westling, G (199la). Integration of sensory information during the programming of precision grip: Comments on the contributions of size cues. Experimental Brain Research, 85, 226-229.

Gordon, A. M., Forssberg, H., Johansson, R. S., \& Westling, G. (1991b). Visual size cues in the programming of manipulative forces during precision grip. Experimental Brain Research, 83, 477-482.

Johansson, R. S., \& Westling, G. (1984). Roles of glabrous skin receptors and sensorimotor memory in automatic control of precision 
grip when lifting rougher or more slippery objects. Experimental Brain Research, 56, 550-564.

Johansson, R. S., \& Westling, G. (1988). Coordinated isometric muscle commands adequately and erroneously programmed for the weight during lifting tasks with precision grip. Experimental Brain Research, 71, 59-71.

JoNEs, L. A. (1986). Perception of force and weight: Theory and research. Psychological Bulletin, 100, 29-42.

Kil breath, S. L., \& Gandevia, S. C. (1991). Independent digit control: Failure to partition perceived heaviness of weights lifted by digits of the human hand. Journal of Physiology, 442, 585-599.

MatThews, P. B. C. (1982). Where does Sherrington's muscular sense originate? Muscles, joints, corollary discharges? Annual Review of Neuroscience, 5, 189-218.

McCullagh, P., \& Nelder, J. A. (1989). Generalized linear models (2nd ed.). London: Chapman \& Hall.

Ross, H. E. (1969). When is a weight not illusory? Quarterly Journal of Experimental Psychology, 21, 346-355.

WEBER, E. H. (1978). The sense of touch (H. E. Ross, Ed. and Trans.). London: Academic Press. (Original work published 1834)

(Manuscript received January 3, 1994; revision accepted for publication October 15, 1994.) 